\title{
Gregory PONTHIÈRE, Économie du vieillissement
}

\section{Alexandra Garabige}

\section{OpenEdition}

\section{Journals}

Édition électronique

URL : https://journals.openedition.org/ress/4095

DOI : $10.4000 /$ ress.4095

ISSN : 1663-4446

\section{Éditeur}

Librairie Droz

\section{Édition imprimée}

Date de publication : 14 décembre 2018

Pagination : 296-298

ISSN : 0048-8046

\section{Référence électronique}

Alexandra Garabige, « Gregory PONTHIÈRE, Économie du vieillissement », Revue européenne des

sciences sociales [En ligne], 56-2 | 2018, mis en ligne le 14 décembre 2018, consulté le 08 janvier 2022. URL : http://journals.openedition.org/ress/4095 ; DOI : https://doi.org/10.4000/ress.4095

Ce document a été généré automatiquement le 8 janvier 2022

(C) Librairie Droz 


\title{
Gregory PONTHIÈRE, Économie du vieillissement
}

\author{
Alexandra Garabige
}

\section{RÉFÉRENCE}

Gregory PONTHIÈRE, 2017, Économie du vieillissement, Paris, La Découverte, « Repères », $128 \mathrm{p}$.

1 Grégory Ponthière s'attèle, dans ce Repères, à l'étude d'un enjeu majeur dans nos sociétés contemporaines: le vieillissement démographique. Il propose d'analyser ce phénomène sous sa dimension économique, c'est-à-dire dans son imbrication avec les conditions matérielles de sa production, à l'aide des grandeurs économiques classiques (consommation, épargne, revenu, etc.). L'ouvrage est structuré autour d'une question centrale - le vieillissement comme facteur de déclin ou de croissance économique et sa prise en charge par les États-providence - et de 5 chapitres synthétisant les théories et approches empiriques de ce champ.

2 Le premier chapitre situe le vieillissement dans une perspective historique et est l'occasion de présenter les différentes définitions et mesures de ce phénomène. L'auteur commence par définir le vieillissement démographique (comme la hausse de la proportion des personnes dites âgées dans la population) et en explique les causes. Phénomène récent, il est lié à la transition démographique, c'est-à-dire au passage d'un régime démographique caractérisé par une mortalité et une natalité élevées à un régime caractérisé par une mortalité et une natalité faibles. Données et graphiques à l'appui (taux de natalité, de moralité, espérance de vie, distribution de la population par âge et sexe), l'auteur caractérise ce phénomène qui affecte différemment les pays en insistant sur le cas de la France. Il expose les causes économiques de cette transition démographique, en éclairant deux facteurs : la baisse de la mortalité et la baisse de la fécondité. Puis, dans une section consacrée aux «définitions et mesures du vieillissement», il explore les différentes dimensions (chronologique, biologique, 
économique et sociale) de ce processus pour en montrer la complexité. L'auteur en conclut que «la quantification du vieillissement est sensible à la conception du vieillissement à laquelle on adhère: âge chronologique, âge biologique, âge économique, âge absolu ou relatif » (p.29).

Le deuxième chapitre, le plus long (30 pages), consacré à la macro-économie du vieillissement, porte sur la question centrale des liens entre vieillissement et croissance et de l'influence réciproque de l'un sur l'autre. Grégory Ponthière annonce d'emblée la nécessité de sortir de la logique "toutes choses égales par ailleurs " pour comprendre les effets du vieillissement sur la croissance. En effet, ce dernier est à l'origine d'une série de mécanismes d'ajustement des comportements en termes d'épargne, d'éducation et de temps de travail qui influent sur la croissance. En retour, le développement économique influe sur le vieillissement. L'auteur expose alors différentes théories économiques et travaux empiriques consacrés au vieillissement tenant compte de l'influence de la population sur l'économie et de l'économie sur la population. Il revient d'abord sur la question du lien entre âge et productivité du travail pour analyser si le vieillissement est défavorable à cette dernière. Il complète ensuite la réflexion par une analyse des modèles de type "cycle de vie ", en montrant par exemple l'impact des comportements d'épargne sur le niveau produit par travailleur ou encore les effets de l'allongement de la durée de vie sur la carrière et sur l'éducation, permettant ainsi de relier décollage économique et transition démographique. L'état des finances publiques, entendu ici à travers le budget des retraites et l'assurance santé, est analysé dans cette même optique. Ce chapitre permet ainsi d'explorer les influences réciproques du vieillissement et de la croissance en tenant compte de divers facteurs influant sur cette dynamique. L'auteur conclut que l'impact du vieillissement sur la croissance économique apparaît ambigu, tant de nombreux effets sont à l'œuvre et jouent dans des directions opposées. Selon ses termes : "Pour résumer, l'effet net du vieillissement sur la croissance dépend des réactions comportementales en termes d'épargne, d'âge de départ à la retraite et d'éducation, réactions qui peuvent contrebalancer - ou pas - la hausse du rapport inactifs/actifs dans la population » (p.64).

4 Dans le troisième chapitre, l'auteur déplace la focale en s'intéressant aux effets du vieillissement non plus sur la croissance mais sur le bien-être, partant du principe que la performance économique d'une société réside dans sa capacité à vaincre les causes de rareté, ici la rareté du temps de la vie humaine. L'auteur insiste d'abord, en s'appuyant sur les travaux pionniers d'Amartya Sen, sur les limites des indicateurs standards de croissance économique, en particulier le PIB, qui ne tiennent pas compte de l'évolution des conditions de survie et donc de la longévité humaine. Il présente ensuite différentes théories économiques qui permettent d'appréhender le vieillissement en termes de bien-être, en particulier l'approche des revenus équivalents qui tient compte de l'espérance de vie et permet de monétiser les gains d'espérance de vie. Ce faisant, l'auteur montre, en s'appuyant sur les résultats de plusieurs travaux d'économistes, l'importance de tenir compte de l'allongement de la durée de vie et de son influence positive sur la performance économique. Il revient également sur les travaux montrant les bénéfices et le rendement des dépenses de santé pour le bien-être des populations, là où elles sont classiquement appréhendées comme un coût.

5 Le quatrième chapitre explore la thématique des inégalités en termes de durées de vie individuelles. La permanence et l'ampleur de ces inégalités sont examinées selon 
l'évolution des probabilités d'atteindre différents âges de la vie. Plusieurs déterminants des inégalités de durée vie sont exposés, travaux d'économistes à l'appui, parmi lesquels : le sexe, le bagage génétique, les modes de vie, l'appartenance ethnique, le niveau d'éducation ou encore le revenu. L'auteur expose ensuite les conséquences économiques des inégalités de longévité en étudiant les relations entre inégalités de durée de vie, richesse et pauvreté. Plusieurs travaux sont présentés qui explorent la question de la mesure de la pauvreté en lien avec la sous-estimation du phénomène (travaux sur les pauvres manquants) et celle des causes de l'accroissement des inégalités de richesse. Dans ce même chapitre, l'auteur s'interroge enfin sur les défis que posent ces inégalités à l'État-providence, en proposant une analyse des conséquences des politiques publiques de distribution dans des économies où les durées de vie sont inégales et en présentant des travaux empiriques, par exemple sur la question de l'âge de départ à la retraite dans les réformes publiques.

Le cinquième chapitre est focalisé sur une autre dimension des inégalités, celles liées à la perte d'autonomie des personnes âgées qui se retrouvent dans l'incapacité d'effectuer des activités quotidiennes. Comme le rappelle l'auteur, la dépendance est un défi majeur dans nos sociétés contemporaines et pose la question de sa prise en charge et de son financement, qu'il s'agisse de l'aide formelle ou de l'aide informelle. Il choisit ensuite de s'interroger sur ce qu'il nomme "le paradoxe de l'assurance dépendance ", à savoir le fait que l'assurance privée dépendance soit si peu répandue malgré l'ampleur du risque et des coûts liés à la dépendance. Plusieurs théories sont exposées pour l'expliquer: du côté de la demande (myopie des personnes âgées, non-achat rationnel ou encore éviction par la famille et l'assurance publique), du côté de l'offre (assurance trop chère, incomplète). La question de la dépendance et des jeux familiaux et celle du rôle de l'État font ensuite l'objet de deux courtes sections spécifiques autour d'une analyse sur l'assurance publique versus l'aide familiale et l'assurance privée.

7 Ainsi, cet ouvrage, sans doute plus accessible à un lecteur déjà familiarisé avec l'approche économique, offre un état des savoirs et des outils de la discipline pour appréhender le vieillissement et ses conséquences (en termes de croissance, de bienêtre ou encore en matière d'inégalité). Pour autant, et comme le souligne l'auteur dans la conclusion, il questionne également les cadres d'analyse de l'économie pour appréhender les défis, tels que la dépendance notamment, qui se posent à l'Étatprovidence.

\section{AUTEURS}

\section{ALEXANDRA GARABIGE}

Paris, INED 